\title{
SOLID STATE MOLECULAR REACTORS IN SPACE
}

\author{
M. Maurette ${ }^{1}$
}

\begin{abstract}
Lunar minerals and impact glasses, convert the polyatomic beam of solar wind ( $\mathrm{SW}$ ) ions into a flux of small molecules (e.g., $\mathrm{H}_{2}$, $\mathrm{N}_{2}, \mathrm{H}_{2} \mathrm{O}, \mathrm{CO}, \mathrm{CO}_{2}, \mathrm{CH}_{4}, \mathrm{C}_{2} \mathrm{H}_{4}, \mathrm{C}_{2} \mathrm{H}_{6}, \mathrm{HCN}$, metal carbides and deuterides, etc.). They thus behave as "Solid State Molecular Reactors". Moreover, $\sim 100-200 \mu \mathrm{m}$ size micrometeoroids ( $\mu \mathrm{Ms}$ ) have also been exposed to the SW in the zodiacal cloud, before being captured by the Earth and recovered as Antarctic micrometeorites. They are mostly composed of a PAH-rich hydrous-carbonaceous material, which amplifies their power as molecular reactors. In particular, during the first $\sim 200$ Myr of the post-lunar period, about $75 \%$ of the $\mu \mathrm{Ms}$ have been melted and/or volatilized upon atmospheric entry. The release of their volatile species triggered a cosmic volcanism around the mesopause that ruled the formation of the early Earth's atmosphere and climate. Furthermore, a fraction of the $\mu \mathrm{Ms}$ that survive unmelted upon atmospheric entry did settle on the proto-oceans floors. Upon further burial in sediments their constituent PAH-rich kerogen was cracked into abiotic oil, which generated giant oil slicks that fed prebiotic chemistry. Many stars, of all ages and types, are embedded into a secondary debris-disk loaded with ion implanted $\mu \mathrm{Ms}$. Some of them are expelled to the interstellar medium (ISM) where they behave first as "dormantinvisible" molecular reactors, until they became reactivated by various processes to synthesize interstellar molecules. This short paper only focus on some highlights of this research dealing with the synthesis of important interstellar molecules, including the most abundant ones $\left(\mathrm{H}_{2}\right.$ and $\mathrm{CO}$ ) and $\mathrm{H}_{2} \mathrm{O}, \mathrm{HCN}$ and PAHs, all involved in prebiotic chemistry.
\end{abstract}

\section{Introduction: Organic geochemistry of lunar molecular reactors}

Lunar dust grains are unique reference samples for learning how to extrapolate the results of laboratory simulation experiments to cosmic dust grains, exposed in ultra-high vacuum $\left(\sim 10^{-13}\right.$ torr $)$ to beams of slow and fast ions. Left panel of

1 CSNSM, University Paris XI, Bâtiment 104, 91405 Orsay-Campus, France 
Figure 1 shows the dark-field electron micrograph of a $\mu$ m-size crystalline lunar feldspar grain, which was observed with a $3 \mathrm{MV}$ high voltage transmission electron microscope by Christian Jouret. Radiation damage features due to the solar wind (SW) and solar energetic particles (SEPs) can be observed, which include -(i)a darker rim with an average thickness of $\sim 40 \mathrm{~nm}$ that decorates an amorphous coating (AC) of SW radiation damage, and -(ii)- a high density of latent tracks of SEPs iron-group nuclei that are best observed as faint lines of dark contrast within the dotted yellow ellipse. The right panel of the same figure shows a zoomed-up detail of another grain, with the contrast enhanced to make the ion tracks more visible.

The identification of these features requires artificial ion implantations of terrestrial lunar analogs with both $\mathrm{SW}$ type ions $(1 \mathrm{keV} / \mathrm{amu})$ and fast Fe ions $\mathrm{E} \sim 1 \mathrm{MeV} / \mathrm{amu}$ ). Upon in-situ thermal annealing in the HVEM, the fossil SW-ACs and SEPs-tracks, as well as the artificial SW-ACs and Fe-tracks, did anneal at the same temperature $\left(\sim 700-800^{\circ} \mathrm{C}\right)$. Moreover, the average thickness of the fossil ACs, of about $40 \mathrm{~nm}$ (see Figs. 1a, 1d, in Maurette 1976), corresponds to both that observed during artificial $1 \mathrm{keV} / \mathrm{amu} \mathrm{SW}$-implants, and to the projected damage range, $\mathrm{R}_{p d} \sim 40 \mathrm{~nm}$, of $1 \mathrm{keV} / \mathrm{amu} \mathrm{SW}$ ions in silicates, as estimated with the SRIM software.

In 1969, organic geochemists discovered that lunar dust grains returned by the Apollo 11 mission did only release small molecules $\left(\mathrm{H}_{2} \mathrm{O}, \mathrm{CO}, \mathrm{CH}_{4}\right.$, and $\mathrm{HCN}$, etc.) during either pyrolysis at moderate temperature $\left(600^{\circ} \mathrm{C}\right)$ or $\mathrm{HF}$ etching. They suggested that these molecules were likely produced during the implantation of high fluences of SW ions in the grains. We next helped testing the validity of this assumption while exposing successively $\sim 1 \mathrm{~cm}^{2}$ flat surfaces of terrestrial analogs (ilmenite, oligoclase and a simulated lunar glass) to high fluence implants $\left(\sim 10^{15}\right.$ to $\left.2 \times 10^{18} \mathrm{~cm}^{-2}\right)$ of "marked" isotopes of hydrogen $\left({ }^{2} \mathrm{D}\right)$, carbon $\left({ }^{13} \mathrm{C}\right)$ and nitrogen $\left({ }^{15} \mathrm{~N}\right)$ ions, at energy of $\sim 1 \mathrm{keV} / \mathrm{amu}$. The group of Al Burlingame (Space Science Laboratory, UC Berkeley) next showed (see Bibring et al. 1974) that the mixture of small molecules released upon heating and/or HF etching from these targets are very similar to the natural blend released from lunar dust grains (Simoneit et al. 1973).

\section{Desorption of molecules during ion implants}

In 1975, Plasma Desorption Mass Spectrometry (see section 4, last paragraph) was already used to show that $\sim 100 \mathrm{MeV}$ fission fragments from ${ }^{252} \mathrm{Cf}$ directly desorb DOH from D-implanted muscovite mica (Maurette et al. 1975). To further investigate this stimulated desorption, another set of the pre-implanted lunar analogs have been exposed to the $10 \mathrm{keV}$ primary beam of Ar of the ionic analyzer built by G. Slodzian. This instrument gives the depth concentration profiles of all species released from the targets. J.P. Bibring observed a large variety of synthesized species ranging from ${ }^{13} \mathrm{C}-\mathrm{Mg}$ to ${ }^{13} \mathrm{C}_{3} \mathrm{D}_{8}$, and including $\mathrm{D}_{2},{ }^{15} \mathrm{~N}_{2}, \mathrm{D}_{2} \mathrm{O}$, and ${ }^{13} \mathrm{CO}$. However, ${ }^{15} \mathrm{NO}$ and ${ }^{15} \mathrm{NO}_{2}$ were not detected $\left({ }^{15} \mathrm{NO} /{ }^{13} \mathrm{CD} \leq 10^{-4}\right)$. 

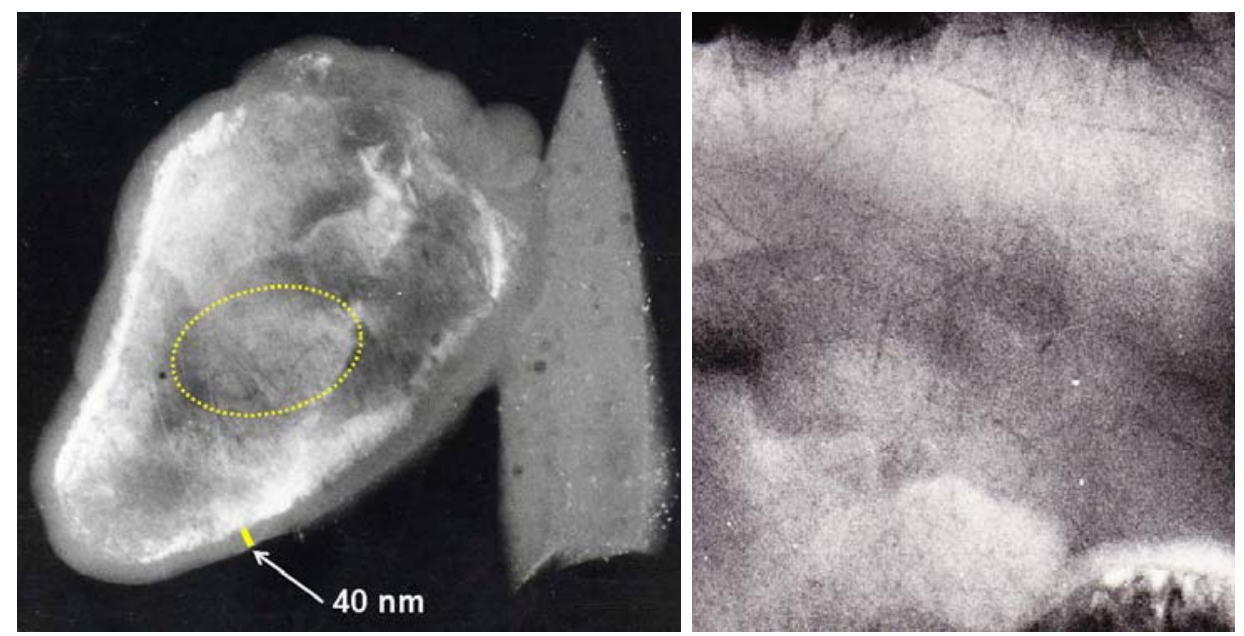

Fig. 1. Left: lunar Micron-size molecular reactor. The shard of impact glass stuck to the main grain has not been noticeably eroded by SW sputtering. Thus it has still not reach the critical saturation SW fluence to function as a molecular reactor. Right: detail of another dust grain with enhanced contrast to make ion tracks more visible to the untrained eye (the thin dark lines in the image).

This study could be extended to a primary beam of $3 \mathrm{keV}$ deuterium, through the decisive help of W. Möller and his team (Max-Plank-Institute für Plasmaphysik, Garching). The three lunar analogs, as well as olivine, silicon, sapphire and a soda-lime glass, have been run with a sophisticated reaction chamber developed for understanding the interactions of a hot DT fusion plasma $\left(\mathrm{T}={ }^{3} \mathrm{He}\right)$ with the first wall of fusion reactors, at $\mathrm{D}$ and $\mathrm{T}$ energy in the $\mathrm{keV}$ to $\mathrm{MeV}$ range. This chamber is equipped with a quadrupole mass spectrometer on-line with both a $30 \mathrm{kV}$ ion accelerator for deuterium implantation, and a 2.5 MV Van de Graaff accelerator for the analysis of the implanted targets with a $750 \mathrm{keV}{ }^{3} \mathrm{He}$ beam, through the $\mathrm{D}\left({ }^{3} \mathrm{He}, \alpha\right) \mathrm{H}$ nuclear reaction. This reaction yields the residual concentration and depth concentration profile of the implanted deuterium. The mass spectrometer was tuned to detect simultaneously the release of $\mathrm{D}_{2}$ and $\mathrm{D}_{2} \mathrm{O}$, which are considered as stumbling blocks in either the gas phase chemistry of the ISM $\left(\mathrm{H}_{2}\right)$ or prebiotic chemistry $\left(\mathrm{H}_{2} \mathrm{O}\right)$.

This on-line study reveals the intimate functioning of the seven molecular reactors (Borgesen et al. 1986). In a first group, including ilmenite, olivine, the alkalipoor LSG $\left(0.02 \% \mathrm{~K}_{2} \mathrm{O}\right)$, sapphire and silicon, a complete retention of D is observed as soon as the deuterium beam is turned on around a fluence of $\sim 10^{15} \mathrm{~cm}^{-2}$. At increasing fluence, a sudden onset of deuterium re-emission $\left(\right.$ as $\left.\mathrm{D}_{2}\right)$ is found around $1 \times 10^{17} \mathrm{~cm}^{-2}$, which finally increases to $80-100 \%$ of the implanted deuterium around a saturation fluence, $\Phi_{s} \sim 10^{18} \mathrm{~cm}^{-2}$. These major characteristics of the deuterium release have also been previously reported for metals (at low 
temperature), and other targets at room temperature, including semiconductors, fused silica and a sintered powder of graphite, which did release both $\mathrm{D}_{2}, \mathrm{CD}, \mathrm{C}_{2} \mathrm{D}$ and $\mathrm{CD}_{4}$. During all the re-emission stage in the O-rich targets, the incoming D yields a $\mathrm{D}_{2} \mathrm{O} / \mathrm{D}$ production ratio of $10^{-4}, 3 \times 10^{-4}$ and $2 \times 10^{-5}$, for ilmenite, oligoclase and the SLG, respectively.

However, both the Na-rich oligoclase and soda-lime glass show a very distinct behavior, as they start loosing (i.e., synthesizing) $\mathrm{D}_{2}$ at the onset of implantation $\left(10^{15} \mathrm{~cm}^{-2}\right)$. They would be the best molecular reactors in a low ion flux environment. On a flat target, perpendicular to the anisotropic SW beam at 1 AU, this fluence is reached in a few months. However, a spherical grain on top of the surface of the Moon would require a longer exposure to accumulate the same fluence on each element of its surface, because the grain is partially shielded from the SW, e.g., during the lunar night.

\section{Hydrous-carbonaceous molecular reactors on the early earth}

In 1975, we already suspected that lunar type molecular reactors might be effective in the ISM ( $c f$. , last section in Maurette \& Price 1975). In 1987, a much more powerful family of $\sim 100-200 \mu \mathrm{m}$ size molecular reactors (i.e., the hydrouscarbonaceous $\mu \mathrm{Ms}$ ) was discovered as Antarctica micrometeorites (see Maurette 2009, for the most recent review about collects and applications). Their marked similarities with the Wild-2 dust particles returned by the Stardust mission in January 2006 (Dobrica et al. 2009), further indicate that they likely originate from comets. This is further supported by IR observations, which revealed that contrarily to previous thoughts, comets are very efficient "dust machine gun" in the solar system (Lisse et al. 2004). Observations of comets P/Encke and P/IRAS with IR telescopes on the ISO and MSX spacecrafts show dust-to-gas mass loss ratios about 50 times larger than those inferred from measurement in the visible. These high mass-loss ratios, which were reconfirmed for comet Temple 1 during the Deep Impact mission, clearly show that comets are the major contributors of dust in the interplanetary medium.

After ejection, most of these $100 \mu \mathrm{m}$ size cometary dust grains have been temporarily stored in the Zodiacal Cloud for duration of $\sim 200000$ yr (Raisbeck \& Yiou 1989), and thus implanted with SW- and SEPs-ions. This cloud is probably a very faint remnant of the secondary debris-disk of the early Sun, which did exponentially decay until about $4 \mathrm{Ga}$ ago, while triggering a period of early heavy bombardment of the Earth-Moon system (Greaves 2005).

About $99 \%$ of the Antarctica micrometeorites are only related to the most volatile-rich hydrous-carbonaceous chondrites, which are relatively rare (about $2-3 \%$ of the meteorite falls). Their major carbonaceous component is a kerogen composed of a mixture of PAH moieties and aliphatic hydrocarbons, and their constituent water (10 wt.\%) is mostly stored in their major hydrous silicate, saponite, that starts dehydrating at $\sim 100^{\circ} \mathrm{C}$. Upon atmospheric entry $\mu \mathrm{Ms}$ would thus release solar wind implanted neon, but also molecules originating from their kerogen $\left(\mathrm{N}_{2}\right.$ and $\left.\mathrm{CO}_{2}\right)$, hydrous silicates $\left(\mathrm{H}_{2} \mathrm{O}\right)$, and metallic sulfides $\left(\mathrm{SO}_{2}\right)$. 
These volatiles ended up making the early Earth's atmosphere (i.e., before the formation of $\mathrm{O}_{2}$ ), during the first $\sim 200 \mathrm{Myr}$ of the post-lunar period, after the giant impact that did form the Moon (Maurette 2009). This impact did also close the formation time interval of the Earth, and blew-off the intractable prelunar atmosphere of the young Earth at a time when the Earth's mantle has already been extensively degassed. Therefore, after the impact, there was not enough volatile species left in the mantle to feed the formation of the massive early Earth's atmosphere, which required the delivery of total amounts of $\mathrm{H}_{2} \mathrm{O}$, $\mathrm{CO}_{2}$ and $\mathrm{SO}_{2}$ corresponding to partial pressures of about 270,60 and 80 bars, respectively (today, $\mathrm{CO}_{2}$ and $\mathrm{SO}_{2}$ are mostly stored as carbonates in the crust and metallic sulfides in the upper mantle, respectively). This $\mu \mathrm{Ms}$ origin of the atmosphere is strongly supported by the $\mathrm{Ne} / \mathrm{N}_{2}$ and the $\mathrm{D} / \mathrm{H}$ ratios of Antarctic micrometeorites. They best fit the corresponding ratios observed in the air and the oceans, respectively, which are incompatible with all other models of atmosphere formation.

Moreover, a fraction of the $\mu \mathrm{Ms}$ that did survive unmelted upon atmospheric entry with their full load of PAH-rich kerogen landed on the proto-oceans floors. They well fit the definition of good "source rocks" for the "economic exploitation" of petroleum, i.e., fine clays with at least $0.5 \mathrm{wt} . \%$ of kerogen (Rondeel 2002). Therefore, they likely generated giant abiotic oil slicks that assisted prebiotic chemistry (Dias \& Maurette 2007). In 1971, Dave Holland and collaborators (Lasaga et al. 1971) proposed a distinct type of primordial oil slick. But their oil was produced during the solar UV irradiation of a primordial methane and nitrogen atmosphere, which had soon to be disregarded.

\section{Kerogen-rich molecular reactors in the ISM}

Frank Shu (1982) pointed out that the extinction of visible starlight could be caused by "bricks". However, he cautioned that bricks cannot produce a reddening of starlight. The combination of true absorption and reddening of this starlight is thus attributed to grains that are slightly smaller $(\leq 0.4 \mu \mathrm{m})$ than the wavelengths of visible light in order to scatter blue light more efficiently than red light. This general extinction does not exclude the presence of $\geq 100 \mu \mathrm{m}$ size "bricklets", which were injected from various stellar sources in the ISM. They could be made of the large diversity of solids that have the potential of desorbing molecules upon ion implants (Sect. 2), including hydrous carbonaceous micrometeoroids (see Sect. 3). All of them would start as somewhat dormant and invisible molecular reactors in the ISM, until they are re-activated by various processes (see next section).

In 1994, 15 Antarctica micrometeorites (i.e., $\mu \mathrm{Ms}$ ) have been ranked on a scale of increasing thermal metamorphism upon atmospheric entry (Clemett et al. 1996). The PAH moieties of their constituent kerogen have been desorbed with the IR laser pulses of a double laser microscope equipped with a reflectron timeof-flight mass spectrometer ( $\left.\mu \mathrm{L}^{2} \mathrm{MS}\right)$. A great diversity of PAH mass spectra were observed, from the simplest spectra of unmelted $\mu \mathrm{Ms}$ (similar to that of the CM2type Murchison chondrite) to the complex spectra of partially melted $\mu \mathrm{Ms}$ that 
mimic that of soot particles (see Figs. 4a to 4d, in Clemett et al. 1996). "Exotic" PAHs were also observed, such as vinyl PAHs that might have promoted interesting organometallic reactions.

McFarlane and co-workers invented the ${ }^{252} \mathrm{Cf}$ plasma desorption mass spectrometer, which allowed the determination of the molecular weight of large organic molecules, such as insulin, which desorbs "intact" from thin deposits irradiated with $\sim 100 \mathrm{MeV}{ }^{252}$ Cf-fission fragments (Torgeson et al. 1974). Therefore, fast ions should also softly desorb PAHs from kerogen, like the IR laser pulses of the $\mu \mathrm{L}^{2} \mathrm{MS}$. We commented (Maurette et al. 2003) on the in-situ FTIR analysis of five organics irradiated with $10 \mathrm{MeV} / \mathrm{amu}{ }^{40} \mathrm{Ar}$ ions, under oxygen free conditions (Reynaud et al. 2001). These materials loose the memory of their initial properties when the radiation dose exceeds $\sim 300$ Mega-Grays, i.e., after an exposure of about $10^{4}$ yr to SEPs at 1 AU. They are all converted into an insoluble material dubbed as kerogen, which might desorb its PAH moieties in the ISM.

\section{Summary and prospects}

In the last $70 \mathrm{yr}$, an abundant literature was published about the main ingredients of dust evolution models in galaxies, including the synthesis of interstellar molecules (cf., Dwek et al. 2009). Our studies show that many reaction channels are opened upon implantation of a polyatomic beam of fast ions in a great diversity of Solid State Molecular Reactors. A challenge is to check whether their easy-to-desorb and abundant species (e.g., $\mathrm{H}_{2}, \mathrm{CO}$ and PAHs), can help simplifying the chemical models proposed for the synthesis of interstellar molecules in the gas-phase, "which contain thousands of reactions, many of which have not been studied theoretically or in the laboratory" (quote from E.Herbst).

Another challenge is to identify the dominant process that can activate molecular desorption from the "dormant" molecular reactors in the ISM. All previous processes, including those listed by Maurette \& Price in 1975, might be fully superseded by the high flux of low energy (2-20 MeV/amu) galactic cosmic rays (LOWs) if McCall and co-workers are right (McCall 2010). They reported challenging papers (e.g., Indriolo et al. 2009) dealing in particular with the determination of the high degree of ionization of the diffuse ISM (as inferred from the $\mathrm{H}_{3}^{+}$ion) and the production rates of $\mathrm{Li}, \mathrm{Be}, \mathrm{B}$ and gamma-rays. They tested multiple LOWs energy spectra and found only two spectra that best fitted the corresponding observations.

I selected their simplest "broken power-law" distribution of cosmic rays in relativistic momentum, reproduced in Figure 2, that represents a differential number flux, $\Phi_{p}$ [particles $\left.\mathrm{cm}^{-2} \mathrm{~s}^{-1} \mathrm{sr}^{-1}(\mathrm{GeV} / \mathrm{n})^{-1}\right]$. Nick Indriolo has kindly computed the corresponding integrated flux of protons, in the three energy ranges considered in this paper: $\Delta_{1}(0.002-1 \mathrm{GeV} / \mathrm{n}) ; \Delta_{2}(2-20 \mathrm{MeV} / \mathrm{n})$ and $\Delta_{3}(5-20 \mathrm{MeV} / \mathrm{n})$. The corresponding "integral" proton fluxes (number $\mathrm{cm}^{-2} \mathrm{~s}^{-1} \mathrm{sr}^{-1}$ ) are about 3.7, 1.5 , and 0.9 , for $\Delta_{1}, \Delta_{2}$, and $\Delta_{3}$, respectively. For grains smaller than the projected range of $2 \mathrm{MeV} / \mathrm{n}$ LOWs in silicates (about $42 \mu \mathrm{m}$ and $18 \mu \mathrm{m}$ for $\mathrm{H}$ and 


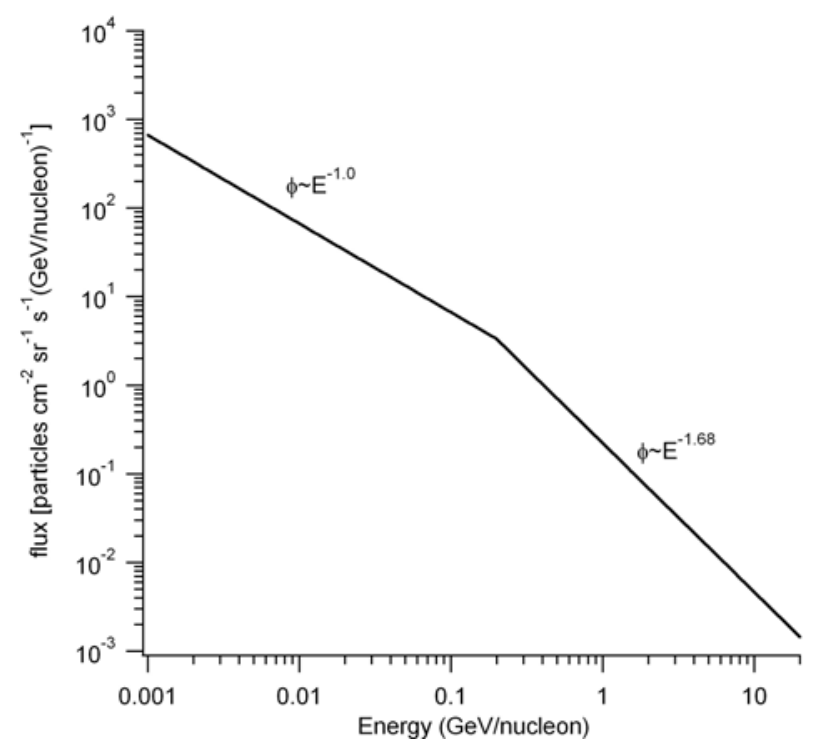

Fig. 2. Possible energy spectrum of low energy cosmic rays in the ISM (Courtesy N. Indriolo).

Fe, respectively), and which include all "visible" grains with sizes $\leq 1 \mu \mathrm{m}$ in the ISM, this value has to be multiplied by $4 \pi$ because the LOWs flux is isotropic.

The lifetime of a large dust grain in the ISM is about $3 \times 10^{7} \mathrm{yr}$, cycling several times between dense and diffuse clouds (see e.g. Tielens 2005). With the resulting flux of 20 protons $\mathrm{cm}^{-2} \mathrm{~s}^{-1}$ (in the $2-20 \mathrm{MeV} / \mathrm{n}$ range) the kerogen simulation experiments discussed in section 4 predict that the organic component of the visible grains would be transformed into kerogen in $\sim 10^{5}$ yr of exposure in the diffuse interstellar clouds. Furthermore, artificial implants of Fe and Ar ions, show that crystalline silicates, would be rendered amorphous by a fluence of $\geq 10^{11} \mathrm{VH} \mathrm{cm}^{-2}$ at the very low temperatures of the ISM, which quench the diffusion induced annealing of atomic defects. Considering a low energy cutoff value of $5 \mathrm{MeV} / \mathrm{n}$ required for the penetration of $\mathrm{VH}$ ions in the diffuse clouds, the Indriolo predicted proton flux $\left(11 \mathrm{~cm}^{-2} \mathrm{~s}^{-1}\right)$ corresponds to an exposure of $\sim 3 \times 10^{7} \mathrm{yr}$ and $\sim 10^{6} \mathrm{yr}$ in the ISM, for a solar $\mathrm{VH} / \mathrm{H}$ ratio $\left(10^{-5}\right)$ and the 30 times higher ratio measured for $\geq 1 \mathrm{GeV} / \mathrm{n}$ GCRs, respectively. A reasonable $\mathrm{VH} / \mathrm{H}$ ratio just a few times higher than solar would insure that in the diffuse ISM, crystalline silicates would be rare but PAHs quite abundant.

The Mcfarlane desorption mass spectrometer can measure PAH desorption yields of kerogen-rich materials, which are required to extrapolate the desorption model to the ISM. The presence of abundant "bricklets" along the orbits of comets is brightly signaled by meteor showers. However, the ISM bricklets have still to be detected. 
I am indebted to: IN2P3 for constant support; A. Burlingame, S. Clemett, C. Hammer, C. Jouret, G. Kurat, W. Möller, N. Indriolo and M. Pourchet for important contributions; E. Dobrica and C. Engrand for their characterization of Antarctic micrometeorites, and; C. Joblin for stimulating thoughts, encouragements and help with LaTeX formatting.

\section{References}

Bibring, J.P., Burlingame, A., Chaumont, J., et al., 1974, Geochim. Cosmochim. Acta, 2, 1747

Borgesen, P., Maurette, M., Möller, W., \& Monart, B., 1986, in Radiation effects in Insulators 3, ed. I.H. Wilson \& R.P. Webb, Gordon and Breach Science Publishers), 711

Clemett, S.J., Chillier, X.D.F., Gillette, S., et al., 1996, Origins life Evol. Biosphere, 28, 425

Dias, F., \& Maurette, M., 2007, Lunar Planet. Sci., 37, A1268

Dobrica, E., Engrand, C., Quirico, E., Montagnac, G., \& Duprat, J., 2009, MAPS, 44, 1643

Dwek, E.E., Galliano, F., \& Jones, A., 2009, ASP Conf. Ser., 414, 183

Greaves, J.S., 2005, Science, 307, 68

Indriolo, N., Fields, B.D., \& McCall, B.J., 2009, ApJ, 694, 257

Lasaga, A.C., Holland, H.D., \& Dwye, M.J., 1971, Science, 174, 53

Lisse C.M., VanCleve, J., Adams, A.C., et al., 2004, Icarus, 171, 444

Maurette, M., \& Price P.B., 1975, Science, 187, 121

Maurette, M., 1976, Nucl. Inst. Meth., 132, 579

Maurette, M, Banifatémi, A., Della-Negra, S., \& Lebeyec, Y., 1983, Nature, 303, 159

Maurette, M., Balanzat, E., \& Duprat J., 2003, Lunar Planet. Sci., 34, A1743

Maurette M., 2009, ASP Conf. Ser., 414, 137

McCall, B.J., 2010, in http://bjm.scs.uiuc.edu/

Raisbeck, G.M., \& Yiou, F., 1989, Meteoritics, 24, A318

Reynaud, J., et al., 2001, Spectrochim. Acta, A57, 797

Rondeel, H.D., 2002, in www.geol.vu.nl

Shu F.H., 1982, The Physical Universe: An Introduction to Astronomy (University Science Books), 1

Simoneit, B.R., Wszolek, P.C., Christiansen, P., Jackson, R.F., \& Burlingame, A.L., 1973, Geochim. Cosmochim. Acta, 37, 1603

Tielens, A.G.G.M., 2005, "The Physics and Chemistry of the Interstellar Medium" (Cambridge University Press), Section 13

Torgeson D.F., Skowronsky, R.P., \& Macfarlane, R.D., 1974, Biochem. Biophys. Res. Commun., 60, 616 